\title{
Distribution Network Fault Locating Based on Limited Measurement Data
}

\author{
Fengde Zhao ${ }^{1}$, Yanqi Zhang ${ }^{2}$, Bangtong Li ${ }^{1}$, Tao $\mathrm{Lin}^{2}$, Rirui Huang', Rusi Chen ${ }^{2}$ \\ ${ }^{l}$ Qionghai power supply bureau, Hainan power grid company, Qionghai China \\ ${ }^{2}$ School of Electrical Engineering, Wuhan University, Wuhan China
}

*Corresponding Author: Tao Lin, School of Electrical Engineering, Wuhan University, Wuhan China

\begin{abstract}
Recent years, power companies pay more and more attention to power quality, and a large amount of money is invested in the power quality monitoring device. Using power quality monitoring device distributed in power system, this paper introduces a distribution network fault locating scheme basing on limited data provided by power quality monitoring device. The scheme can not only achieve fault locating taking advantage of power quality monitoring device, but also increase maintenance efficiency.
\end{abstract}

Keywords: Fault locating; Distribution network; Measurement data

\section{INTRODUCTION}

At present, basing on the power grid topology, and operation mode information, and the limited measurement information provided by power distribution automation or power quality monitoring system, it is a hot spot to realize the accurate positioning of electrical fault points in the distribution network $^{[1]}$. In this paper, we will introduce a fault locating scheme based on the limited data in the distribution network. The scheme ascertains the candidate fault area and electrical fault types according to the observation value of current and voltage at the limited observation point before and after the failure firstly, further realizes the fault locating which is strong support to grid security, stability and reliable operation ${ }^{[2-3]}$.

Traditional fault locating methods, such as Impedance method and Travel wave method ${ }^{[4]}$, achieve good effect when applied to fault locating of transmission network. However, the distribution network has complex structure, lots of branches, insufficient transformer and less fault locating devices, difficulties occur when apply those methods to accurate fault locating of distribution network. Although fault principle of small current grounding system are studied, but the existing fault locating methods of distribution network not only need to install a large number of voltage and current transformer, high precision data collectors for acquiring transient zero sequence component, but also only the fault section can be located ${ }^{[5-6]}$. In order to save the cost of the power grid, and to accurately locate the distribution network fault, a fault locating scheme based on the limited data in the distribution network will be introduced.

\section{OVERALl SChEME OF ACCURATE FAUlt Locating}

The proposed fault locating scheme contains four steps. Firstly, determining the moment of power grid failure before locating in order to distinguish the data before and after the failure. Secondly, Judging the fault type according to the three phase voltage phase information of the monitoring points. Thirdly, based on the judgment of the upstream and downstream of the fault source, obtaining the collection of possible fault lines or bus lines, and the candidate area of the fault can be located. Finally, building the optimization model based on the short-circuit calculation, and obtaining the fault distance and fault resistance by solving the above model. Thus the accurate fault locating is realized. 


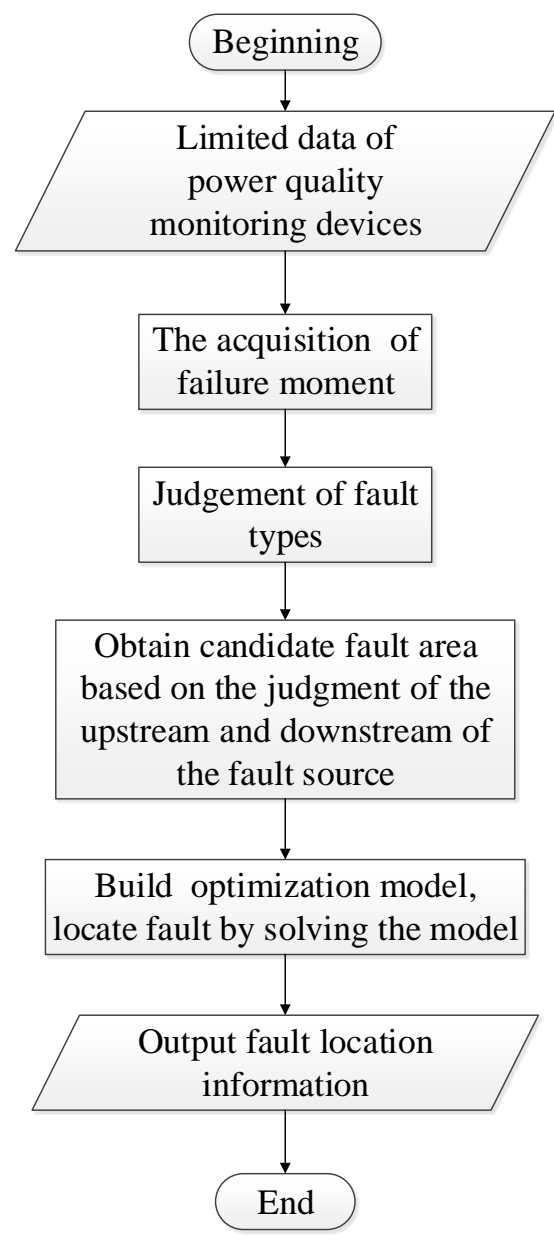

Fig1. Steps of fault locating scheme

\section{Detection of Fault Occurance}

The locating scheme aims at locating the distribution network fault based on the limited measurement data. The scheme relies on recorded current and voltage information measured by power quality monitoring device of limited nodes to locate. Before locating, what need to do first is to determine the moment of power grid failure so as to distinguish the data before and after fault locating conveniently.

When the power system has a short circuit fault, there is basic phenomenon that the current increases dramatically. In some conditions, the maximum instantaneous value of the current can be up to 10 to 15 times the rated current. Besides, while the current increases dramatically, the voltage in the system will drop considerably. For example, when a three-phase short circuit occurs, the voltage of the short circuit point will be reduced to zero, and the voltage of each point near the short circuit point will be significantly reduced ${ }^{[7]}$.

It can be concluded that when the proposed scheme locates, the current and voltage measured by the power quality monitoring device of the limited node can be changed greatly if a short-circuit fault occurs in the monitored distribution network. That is to say, we can acquire the fault moment by monitoring changing of the current and voltage measured by the power quality monitoring device of the limited node.

\section{JudGEMENT OF FAULT TyPe}

The closer the measuring point is to the point of failure, the lower the voltage amplitude is, the more it can represent the actual situation of the fault point. So when determining the fault type, the lowest monitoring point voltage in the three phase voltage amplitude of each monitoring point should be extracted as a basis of judgment firstly. Then, calculate the mean value of the three-phase voltage amplitude of this monitoring point $U_{m}$, and determine the fault type by comparing the magnitude of the three-phase voltage amplitude with the mean value and monitoring zero sequence component information. The criterion is as follows: 
1) If the fault type is single-phase earth fault, there will be characteristic that the voltage amplitude of one phase is lower than the mean value, while the other two phase voltage amplitude is higher than the mean value, and the difference between the three-phase voltage amplitude and the mean value should be greater than that of a certain threshold $p_{u}$.

2) If the fault type is phase-phase earth fault or phase-phase fault, there will be characteristic that the voltage amplitude of the two phases is below the mean value, while the other phase voltage amplitude is higher than the mean value, and the difference between the three-phase voltage amplitude and the mean value should be greater than that of a certain threshold $p_{u}$. Besides, for phase-phase earth fault the zero sequence component should be greater than one value $p_{u 0}$, and for phase-phase fault the zero sequence component should be less than one value $p_{u 0}$.

3) If the fault type is three phase earth fault, there will be characteristic that the difference between the three-phase voltage amplitude and the mean value should be less than a certain threshold $p_{u}$.

Since the three-phase voltage amplitude of the symmetric fault in the actual system cannot be identical, it is necessary to set a threshold to determine the symmetric fault. As long as the magnitude of the three-phase voltage deviation is less than the threshold value, it can be considered that it is a symmetric fault, whereas, it is an asymmetric fault. That is to say, in the case of asymmetric failure, the magnitude of the three-phase voltage deviation of the three phase voltage deviation must be larger than that of symmetrical failure.

The difference between phase-phase fault and phase-phase earth fault is whether there are zero sequence components. Due to the existence of an ungrounded system in the actual system, the zero sequence components of the phase-phase earth fault can also be very small. So the threshold of the zero sequence component $p_{u 0}$ is sometimes very small.

\section{Obtaining Candidate Fault Area}

This scheme determines the upstream and downstream relations of the fault source relative to each monitoring point using the method proposed by reference [8]. Then narrow down the range of the fault area according to relative position and grid topology.

The reference [8] thinks of the power side and the load side as a whole separately. The reference analyzes the equivalent impedance of the non-disturbed side by analyzing change of voltage and current during failure, and determines the upstream and downstream relations of the fault source relative to each monitoring point by the symbol of the real part of the equivalent impedance. The following is a brief description.

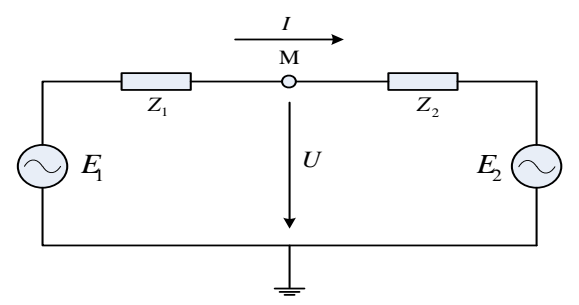

Fig2. Equivalent circuit of fault point detection and analysis

As shown in figure 2 , it is assumed that the user's side fails and causes voltage drop at the measurement point $\mathrm{M}$. There will be:

$\mathrm{Z}_{e}=\frac{\Delta U}{\Delta I}=\frac{U_{\text {during }}-U_{\text {pre }}}{I_{\text {during }}-I_{\text {pre }}}$

In the formula, $\left(U_{p r e}, I_{p r e}\right)$ and $\left(U_{d u}, I_{d u}\right)$ are the basic-sequence voltage and current in the fault process before and during the fault.

The reference [8] proposed that the upstream and downstream relations of the fault source relative to each monitoring point can be determined according the $\operatorname{real}\left(\mathrm{Z}_{e}\right)$. The specific rules of judgment are 
as follows:

(1) If $\operatorname{real}\left(\mathrm{Z}_{e}\right)>0$, the relative relation is upstream;

(2) If $\operatorname{real}\left(\mathrm{Z}_{e}\right)<0$, the relative relation is downstream;

Determine the relative relation between the fault point and the limited monitoring points according to the above criteria. Then carry out comprehensive analysis based on network topology and relative position of monitoring points. And candidate fault area can be obtained.

\section{AcCurate Fault Locating}

In the case of a known candidate fault area and fault type, it is the key to the fault locating to calculate fault distance which fault resistance is related. The short circuit calculation is the basis of analyzing fault resistance and fault distance, so the short circuit calculation method is introduced firstly.

\subsection{Short Circuit Calculation Method}

At present, the computer algorithm of short-circuit calculation is more mature. The complete equipment parameter information can be provided by the safety production management information system and equipment management system, and the voltage data of the monitoring point can be obtained directly from the power quality monitoring system. Therefore, use the sequence component method to calculate the voltage of the node under different fault types. In the sequence component method, the voltage and current relationship of each sequence component is shown in formula (2) ${ }^{[9]}$.

$$
\left\{\begin{array}{l}
\dot{U}_{m i}^{(1)}=\dot{U}_{m i}^{|0|}-Z_{m i, f}^{(1)} \dot{I}_{f}^{(1)} \\
\dot{U}_{m i}^{(2)}=-Z_{m i, f}^{(2)} \dot{I}_{f}^{(2)} \\
\dot{U}_{m i}^{(0)}=-Z_{m i, f}^{(0)} \dot{I}_{f}^{(0)}
\end{array}\right.
$$

In the formula, the superscript (1)、(2)、(0) indicate positive, negative and zero sequence; the $\dot{U}_{m i}^{|0|}$ indicates the voltage of the monitoring point i before the failure; the $Z_{m i, f}$ indicates the mutual impedance between the monitoring point $\mathrm{i}$ and the fault point $\mathrm{f}$; the $I_{f}$ indicates the fault current.

\subsection{Accurate Fault Locating Model}

In order to accurately locate the fault, this scheme establishes the optimization model of fault locating in the case of that the error of the actual observed value and the theoretical calculation value is minimal.

The fault is located based on the voltage information recorded by the limited power quality monitoring device. In particular, the fault locating model is established in the case of that the actual observations and theoretical calculations of each phase voltage $U_{m, a} 、 U_{m, b} 、 U_{m, c}$ are minimal, and that the fault distance $\mathrm{p}$ and fault resistance $z_{f}$ are optimized variables. The fault locating model is as follows:

$$
\min J\left(p, z_{f}\right)=\sum_{m=1}^{q}\left|U_{m}-U_{m}\left(p, z_{f}\right)\right|
$$

In the formula, the constraint condition is $0 \leq p \leq 1, z_{f} \geq 0$; the $U_{m}$ indicates the actual voltage of the observation point $\mathrm{m}$; the $\mathrm{q}$ indicates the total number of monitoring points; the $\dot{U}_{m i}$ indicates the voltage of the observation point I; the $U_{m}\left(p, z_{f}\right)$ indicates theoretical calculation of voltage; The absolute value is the modulus of the complex number.

After establishing the accurate locating optimization model, the accurate fault distance $\mathrm{p}$ and fault resistance $z_{f}$ can be obtained by solving the above model. 


\section{CONCLUSION}

Nowadays users pay more and more attention to power quality, so it is necessary to adopt simple and reliable fault locating technique in distribution network. In this paper, A fault locating scheme will be introduced based on the limited data in the distribution network. The scheme ascertains the fault location according to the observation value of current and voltage at the limited observation point before and after the failure, and it helps to improve the efficiency of maintenance in distribution network.

\section{ACKNOWLEDGEMENT}

The authors would like to acknowledge gratefully the support of Qionghai Power Supply Bureau, China for Research and Application of Distribution Network Fault Locating Technology Based on limited Measurements (070400kk52170001).

\section{REFERENCES}

[1] ZHANG Yi, LIN Yan, WU Danyue. Current Status and Development Trend of Power Quality Monitoring System[J]. Power System Protection and Control, 2015, (02): 138-147.

[2] PENG Jinning. Study of Reliability Assessment for Distribution Systems[D]. Xi'an: Xi'an University of Technology, 2004.

[3] Zhuomin Zhou, Asayoshi Ishida, Etsuhiko Maeda, etal. Capacity Planning and Practicality Evaluation of Grid-Independent Power System Based on Supply Reliability[J].Electrical engineering in Japan, 2012, 81(2): 1-9.

[4] LU Jiping, LI Yin, LI Jian, etal. A new method of single-end fault ranging based on the travelling wave method and impedance method[J]. Automation of Electric Power System, 2007, (23): 65-69.

[5] ZHANG Linli. Study on Fault Locating Method in Non-effectively Grounded Network and Its Application[D]. Shandong University, China, 2013.

[6] Gong Y, Guzman A. Integrated Fault Locating System for Power Distribution Feeders[J].IEEE Transactions on Industry Applications, 2013, 49(3): 1071-1078.

[7] ZHENG Rihong, GU Xiufnag, HAN Ruyue, etal. Analysis of Short Circuit Faults of Distribution Network and Research of Its Identification Methods[J]. Industry and Mine Automation, 2012, (04): 23-29.

[8] Tayjasanant Thavatchai, Li Chun, XuWilsun. Resistance sign-based method for voltage sag source detection[J]. IEEE Trans on Power Delivery, 2005, 20(4): 25-44.

[9] ZHOU Liancheng, LUO Aiqing, WANG Qianggang, etal. Short Circuit Calculation of Symmetrical Component Method in Distribution Network with Induction generators[J]. Automation of Electric Power System, 2013, (11): 65-70.

\section{AUTHORS' BIOGRAPHY}

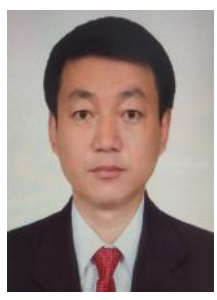

Fengde Zhao, was born in 1966. His native place is the city of Mishan, Heilongjiang Province, China. He has a bachelor's degree and is a senior engineer.

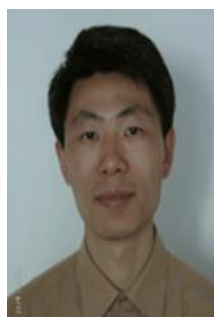

Tao Lin, is a professor of school of electrical engineering at Wuhan University, China. His research fields are power system relaying, power quality, power system operation.

Citation: Tao Lin et al.(2017). Distribution Network Fault Locating Based on Limited Measurement Data, International Journal of Research Studies in Electrical and Electronics Engineering (IJRSEEE), 3(4), pp.5-9, DOI: http://dx.doi.org/10.204 31/2454-9436.0304002.

Copyright: (C) 2017 Tao Lin et al. This is an open-access article distributed under the terms of the Creative Commons Attribution License, which permits unrestricted use, distribution, and reproduction in any medium, provided the original author and source are credited 